A10

doi: 10.14232/ syrpharmacognosy.2021.a10

\title{
Phytochemical analysis of three food plants
}

\section{Klára Horváth-Boros}

Email: horvath-boros.klara@szte.hu

Although the nutritive value of food plants depends on primary metabolites such as carbohydrates, lipids and proteins, their physiological effects and enjoyment values largely depend on secondary metabolites. The aim of our work was to analyse the secondary metabolites of three food plants. The examined species included Camellia sinensis, the source of the most widely consumed herbal beverage, Coffea sp., that are used not only to prepare coffee but also to produce weight loss supplements, and Capsicum annuum, a characteristic spice of Hungarian gastronomy.

In case of $C$. sinensis, we first developed a densitometric method to measure the amount of theanine in tea. The advantage of this method is that it is fast, reliable and does not require special instrumentation [1]. Subsequently, a reversed-phase HPLC method was developed to examine the theanine content of tea leaves [2]. Moreover, this robust method is suitable for the simultaneous determination of the stimulant caffeine and the sedative theanine [3].

The analysis of coffee samples focused on the chlorogenic acid content, a group of compounds that have confirmed weight reducing effect. Our method allows the simultaneous determination of caffeine and chlorogenic acid. Further, a reversed-phase HPLC method was developed for the quantitative measurement of capsaicin analogues in peppers.

We have shown that the theanine content of tea does not always correlate with the degree of fermentation, since the theanine content of some green tea samples were below the levels of black teas [3]. In case of green coffee samples, we identified varieties with high chlorogenic acid content and developed a method that allows the removal of caffeine to enrich chlorogenic acids. The rationale of the Capsicum experiments was to find correlation with the organoleptic characteristics to contribute to the development of quality standards based on objective data.

\section{Supervisor: Dezső Csupor}

\section{Acknowledgements:}

This work was supported by the ÚNKP-20-3 - New National Excellence Program of the Ministry for Innovation and Technology from the source of the National Research, Development and Innovation Fund.

\section{References}

[1] Dezső Csupor et al. Journal of Planar Chromatography 2012; 25(6):571-574.

[2] Dezső Csupor et al. Food Anal. Methods 2014; 7(3):591-596.

[3] Klára Boros et al. Pharmacogn Mag. 2016; 12(45):75-79. 